\title{
ON AN ALGEBRAIZATION OF THE RIEMANN-HURWITZ RELATION
}

\author{
By IZUMI KURIBAYASHI
}

\section{Introduction.}

In this paper we study the canonical representation $\operatorname{Aut}(M) \rightarrow G L(g, \boldsymbol{C})$ with the space of holomorphic differentials on $M$ as its representation module, where $M$ is a compact Riemann surface of genus $g \geqq 2$ (cf. (1.1)). For an automorphism group $A G$ of $M$ we denote its image by $R(M, A G)$. The $G L(g, C)$-conjugate class of $R(M, A G)$ appears as an invariant of the holomorphic family of Riemann surfaces which is defined by the subgroup of Teichmüller modular group corresponding to the pair $(M, A G)$ (cf. [4], [5]). From such a point of view among others we consider it a problem to determine $R(M, A G)$ 's.

In this paper we introduce two necessary conditions, which turn out (in $\S 2$ ) sufficient in case $g=2$, for a finite subgroup $G$ of $G L(g, C)$ to be conjugate to some $R(M, A G)$. In $\S 1$ we make an algebraic formulation of the RiemannHurwitz relation, in terms of which one of our conditions is given. In fact we define the data of "ramification" for a (special type of) finite subgroup of $G L(g, C)$ and we show our formulation is valid in this case. In $\S 2$ we introduce another condition on $G$ that the character defined by $G$ is of the form of the Eichler trace formula. It is known [6] that this condition is also sufficient in case where $G$ is of prime order (and $g \geqq 2$ ). Using these two conditions, we determine 21 types of representatives (up to $G L(g, C)$-conjugacy) of $R(M, A G)$ 's in the case $g=2$.

In a similar line we shall determine $R(M, A G)$ 's in another place when $g=3$ (55 types) and $g=4$ (74 types).

\section{Notation.}

As usual $\boldsymbol{C}$ mean the field of complex numbers. The subgroup of a group generated by a family $\left\{A_{1}, \cdots, A_{r}\right\}$ of its elements is denoted by $\left\langle A_{1}, \cdots, A_{r}\right\rangle$. We write $\# X$ for the cardinality of a finite set $X$. And for an element $A$ of a group we denote its order by $\# A$. If $T$ is an element of $G L(g, C), T^{*}$ denotes the automorphism of $G L(g, \boldsymbol{C})$ sending $A$ to $T^{-1} \cdot A \cdot T(A \in G L(g, \boldsymbol{C}))$.

Recelved August 17, 1983 


\section{$\S 1$. Riemann-Hurwitz relation.}

In this section we use the following notation for a group $G$ and its subgroup $H$.

$$
\begin{aligned}
& C Y(G)=\{K \mid K \text { is a nontrivial cyclic subgroup of } G\}, \\
& C Y(G \mid \supseteqq H)=\{K \in C Y(G) \mid K \text { contains } H\}, \\
& C Y(G \mid \supseteqq H)=\{K \in C Y(G) \mid K \text { contains } H \text { strictly } .
\end{aligned}
$$

1.1. Motivation. Let $M$ be a compact Riemann surface of genus $g \geqq 2$, and let $A G$ be an automorphism group of $M$. For each point $P$ of $M$ we denote by $A G(P)$ the stabilizer of $A G$ at $P$. It is noted that $A G(P)$ is a cyclic group (see e.g. [3], III. 7.7.). For each nontrivial cyclic subgroup $H$ of $A G$, we define as follows.

$$
\begin{aligned}
& r(H)=\#\{P \in M \mid A G(P) \text { contains } H\}, \\
& r_{*}(H ; A G)=\sharp\{P \in M \mid A G(P)=H\}, \\
& l(H ; A G)=\# \pi(\{P \in M \mid A G(P)=H\}),
\end{aligned}
$$

where $\pi$ denotes the natural mapping of $M$ onto $M / A G$.

Here we recall the Riemann-Hurwitz relation for $\pi$ (see e.g. [3], V. 1.3.):

$$
2 g-2=n\left(2 \cdot g_{0}(A G)-2\right)+n \sum_{\imath} l\left(H_{2} ; A G\right) \cdot\left\{1-\left(1 / \# H_{\imath}\right)\right\},
$$

where $n=\# A G, g_{0}(A G)$ denotes the genus of $M / A G$, and $\left\{H_{i}\right\}$ is a set of representatives of the $A G$-conjugacy classes of $C Y(A G)$. And we note the following facts (1) and (2).

$$
r_{*}(H ; A G)=r(H)-\sum_{K} r_{*}(K ; A G),
$$

where $K$ ranges over the set $C Y(A G \mid \supsetneq H)$.

$$
l(H ; A G)=r_{*}(H ; A G) /\left[N_{A G}(H): H\right] .
$$

where [:] denotes the index and $N_{A G}(H)$ denotes the normalizer of $H$ in $A G$.

Let $R:$ Aut $(M) \rightarrow G L(g, C)$ denote the canonical representation for a (fixed) basis $\left\{\xi_{1}, \cdots, \xi_{g}\right\}$ of holomorphic differentials on $M$. In fact, the matrix $R(\sigma)=$ $\left(s_{i j}\right)$ (corresponding to a $\sigma \in \operatorname{Aut}(M)$ ) is defined by the relation :

$$
\sigma^{*}\left(\xi_{\imath}\right)=\sum_{j=1}^{g} s_{\imath}, \xi, \quad(l=1, \cdots, g) .
$$

It is noted that $R$ is faithful (see e.g. [3], V. 2.1.). Then it is easy to see the following facts (3) and (4).

$$
r(H)=2-\left\{\operatorname{Tr}(R(\sigma))+\operatorname{Tr}\left(R(\sigma)^{-1}\right)\right\} \quad \text { if } \quad H=\langle\sigma\rangle
$$


(Lefschetz fixed point formula, see e.g. [3], V. 2.9.).

(4) $g_{0}(A G)$ coincides with the dimension of the $R(M, A G)$-invariant subspace of the $\boldsymbol{C}$-vector space $\boldsymbol{C} \times \cdots \times \boldsymbol{C}$ ( $g$-times) (under the natural action) (cf. [3], V. 2.2.).

1.2. Algebraization. We are motivated by the facts in (1.1) to consider its algebraization using matrices as follows.

Definition. For a matrix $A$ of $G L(g, C)$ of finite order, we say that $A$ satisfies $\left(E_{0}\right)$, if $\operatorname{Tr}(A)+\operatorname{Tr}\left(A^{-1}\right)$ is an integer, or equivalently, if the relation

$$
\operatorname{Tr}(A)+\operatorname{Tr}\left(A^{-1}\right)=\operatorname{Tr}\left(A^{k}\right)+\operatorname{Tr}\left(A^{-k}\right)
$$

holds for each integer $k$ such that $(k, \# A)=1$. For a finite subgroup $G$ of $G L(g, C)$, we say that $G$ satisfies $\left(E_{0}\right)$, if each element of $G$ satisfies $\left(E_{0}\right)$.

Definition. Let $G$ be a finite subgroup of $G L(g, C)$ which satisfies $\left(E_{0}\right)$, and let $H$ be a nontrivial cyclic subgroup of $G$. Then we define as follows.

$$
\begin{gathered}
g_{0}(G)=(1 / \mathbb{\#} G) \cdot \sum_{A \in G} \operatorname{Tr}(A), \\
r(H)=2-\left\{\operatorname{Tr}(A)+\operatorname{Tr}\left(A^{-1}\right)\right\}, \quad \text { where } H=\langle A\rangle, \\
r_{*}(H ; G)=r(H)-\sum_{K} r_{*}(K ; G),
\end{gathered}
$$

where $K$ ranges over the set $C Y(G \mid \supsetneq H)$. To state more precisely, $r_{*}(H ; G)$ is defined by the descending induction on $H$ with respect to the inclusion relations in $C Y(G)$.

$$
l(H ; G)=r_{*}(H ; G) /\left[N_{G}(H): H\right] .
$$

For the sake of brevity we set $r(\langle A\rangle)=r(A), \quad r_{*}(\langle A\rangle ; G)=r_{*}(\langle A\rangle)=r_{*}(A)$ and $l(\langle A\rangle ; G)=l(\langle A\rangle)=l(A)$, for each element $A(\neq I)$ of $G$. (It is convenient to define $l(\langle I\rangle ; G)=l(\langle I\rangle)=l(I)$ as 0 , as the case may be. $)$

Remark. (i) The number $r(H)$ is well-defined by virtue of $\left(E_{0}\right)$.

(ii) By the orthogonality relations for characters, it is noted that $g_{0}(G)$ coincides with the dimension of the $G$-invariant subspace of $\boldsymbol{C} \times \cdots \times \boldsymbol{C}$ ( $g$-times) (under the natural action).

(iii) If $G=R(M, A G)$, then the notions $r, r_{*}, l$ and $g_{0}$ in (1.1) and (1.2) are compatible (via the representation).

\subsection{Riemann-Hurwitz relation.}

Proposition 1. If $G$ is a finite subgroup of $G L(g, C)$ which satisfies $\left(E_{0}\right)$, then we have the following relation: 
$(R H)$

$$
2 g-2=n\left(2 \cdot g_{0}(G)-2\right)+n \cdot \sum_{\imath} l\left(H_{2} ; G\right)\left\{1-\left(1 / \# H_{\imath}\right)\right\},
$$

where $n=\sharp G$ and $\left\{H_{i}\right\}$ is a complete set of representatives of the G-conjugacy classes of $C Y(G)$.

Proof. From the definition of $g_{0}(G)$ :

$$
g_{0}(G)=(1 / n) \cdot \sum_{A \in G} \operatorname{Tr}(A)
$$

it follows that

$$
2 n \cdot g_{0}(G)=2 \cdot \operatorname{Tr}(I)+\sum_{A \in G^{\times}}\left\{\operatorname{Tr}(A)+\operatorname{Tr}\left(A^{-1}\right)\right\},
$$

where $G^{\times}$denotes the set $G \backslash\{I\}$. Since $\operatorname{Tr}(I)=g$ and $r(A)=2-\left\{\operatorname{Tr}(A)+\operatorname{Tr}\left(A^{-1}\right)\right\}$, we have that

$$
2 g-2=n\left(2 \cdot g_{0}(G)-2\right)+\sum_{A \in G^{\times}} r(A) .
$$

Thus it suffices to show that

$$
\sum_{A \in G^{\times}} r(A)=n \sum_{\imath} l\left(H_{\imath}\right)\left\{1-\left(1 / n_{\imath}\right)\right\},
$$

where each $n_{\imath}$ denotes the number $\sharp H_{\imath}$.

For each $\imath$, let $\left\{H_{i k} \mid k=1, \cdots, k_{\imath}\right\}$ be the $G$-conjugacy class of $H_{\imath}=H_{\imath 1}$, where $k_{\imath}=\left[G: N_{G}\left(H_{\imath}\right)\right]$. To prove $(+)$ first we note by the definition that

$$
l\left(H_{\imath}\right)=r_{*}\left(H_{\imath}\right)\left[G: N_{G}\left(H_{\imath}\right)\right] /\left[G: H_{\imath}\right]=r_{*}\left(H_{\imath}\right) \cdot k_{\imath} \cdot n_{i} / n .
$$

And hence we see that

$$
\begin{aligned}
n \cdot l\left(H_{\imath}\right)\left\{1-\left(1 / n_{\imath}\right)\right\} & =r_{*}\left(H_{\imath}\right) \cdot k_{i}\left(n_{\imath}-1\right) \\
& =k_{\imath}\left\{\sum_{A \in H_{i}} r_{*}(A)+\sum_{A \in H^{*}} r_{*}\left(H_{\imath}\right)\right\} \\
& =\sum_{k=1}^{k_{l}}\left\{\sum_{A \in H_{\imath} k^{\circ}} r_{*}(A)+\sum_{A \in H_{\imath k^{*}}} r_{*}\left(H_{\imath k}\right)\right\},
\end{aligned}
$$

where, for a subgroup $H$ of $G, H^{\circ}$ (resp. $\left.H^{*}\right)$ denotes the set $\{A \in H \mid\langle A\rangle=H\}$ (resp. $\left\{A \in H^{\times} \mid\langle A\rangle \neq H\right\}$ ). Secondly we note that

$$
\begin{aligned}
& \sum_{\imath} \sum_{k=1}^{k_{2}} \sum_{A \in H_{\imath k^{\circ}}} r_{*}(A)=\sum_{A \in G^{\times}} r_{*}(A) \\
& \sum_{\imath} \sum_{k=1}^{k_{\imath}} \sum_{A \in H_{\imath k^{*}}} r_{*}\left(H_{\iota k}\right)=\sum_{A \in G^{\times}} \sum_{H_{A}} r_{*}\left(H_{A}\right),
\end{aligned}
$$

where $H_{A}$ ranges over the set $C Y(G \mid \supseteqq\langle A\rangle)$. Hence we obtain

$$
\begin{aligned}
\sum_{\imath} n \cdot l\left(H_{\imath}\right)\left\{1-\left(1 / n_{\imath}\right)\right\} & =\sum_{A \in G^{\times}}\left\{r_{*}(A)+\sum_{H_{A}} r_{*}\left(H_{A}\right)\right\} \\
& =\sum_{A \in G^{\times}} r(A),
\end{aligned}
$$


as desired (where $H_{A}$ ranges as above).

Q.E. D.

1.4. $\left(\boldsymbol{R} \boldsymbol{H}_{+}\right)$. We introduce a "necessary" condition.

Definition. We say that a finite subgroup $G$ of $G L(g, C)$ satisfies $\left(R H_{+}\right)$ if $G$ satisfies $\left(E_{0}\right)$ and if $l(H ; G)$ is a non-negative integer for any nontrivial cyclic subgroup $H$ of $G$.

In this case, letting $\left\{H_{1}, \cdots, H_{s}\right\}$ be a set of representatives of the $G$-conjugacy classes of $C Y(G)$, we define the "RH data" of $G, R H(G)$, as follows :

$$
R H(G)=[\# G, g_{0}(G) ; \underbrace{n_{1}, \cdots, n_{1}}_{l\left(H_{1}\right) \text {-times }}, \cdots, \underbrace{n_{s}, \cdots, n_{s}}_{l\left(H_{s}\right) \text {-times }}]
$$

where $n_{\imath}=\sharp H_{\imath}(\imath=1, \cdots, s)$. Here we may always assume that $n_{1} \leqq n_{2} \leqq \cdots \leqq n_{s}$.

Remark. If $G=R(M, A G)$ then $G$ satisfies $\left(R H_{+}\right)$(cf. (1.1) and (1.2)).

We shall mention two corollaries of Proposition 1.

COROllary 2. Let $G$ be a finite subgroup of $G L(g, C)$ (where $g \geqq 2$ ) which satisfies $\left(\mathrm{RH}_{+}\right)$. Then we have the following.

(1) $\sharp G \leqq 84(g-1)$.

(2) If $\# G>4(g-1)$, then $g_{0}(G)=0$.

Proof. The argument for (1) (resp. (2)) is almost identical to [3], V. 1.3. (resp. [1], Lemma 8).

COROLlary 3. Let $G$ be a finte subgroup of $G L(g, C)$ which satisfies $\left(R H_{+}\right)$. Let $d(G)$ denote the integer

$$
3 \cdot g_{0}(G)-3+\sum_{\imath} l\left(H_{2} ; G\right),
$$

where $\left\{H_{\imath}\right\}$ is as in the above definition. Then $d(G)$ is nonnegative.

Proof. We may assume that $g_{0}(G)=0$. We wish to show that $\Sigma l\left(H_{\imath}\right) \geqq 3$, so suppose $\sum l\left(H_{\imath}\right) \leqq 2$. Then it follows from Proposition 1 that

$$
2 g-2 \leqq-2 n+n\left\{\left(1-\left(1 / n_{1}\right)\right)+\left(1-\left(1 / n_{2}\right)\right)\right\}
$$

for some (positive) divisors $n_{\imath}$ of $n(\imath=1,2)$. This means that $g$ is smaller than 1 , which is absurd.

Q.E.D.

1.5. We prove a proposition which shall be used for the classification of $R(M, A G)^{\prime}$ 's. In this numero we assume that $G$ is a finite subgroup of $G L(g, C)$ and $G^{\prime}$ is a subgroup of $G$.

Proposition 4. If $G$ satısfies $\left(R H_{+}\right)$, then $G^{\prime}$ also satısfies $\left(R H_{+}\right)$.

Before giving its proof, we insert two lemmas. 
Lemma 5. Let $H$ be a cyclic subgroup of $G$, and let $N\left(G \mid H, G^{\prime}\right)$ denote the set $\left\{T \in G \mid T^{*}(H) \cap G^{\prime}=H \cap G^{\prime}\right\}$, Then $\# N\left(G \mid H, G^{\prime}\right)$ is divusible by $\sharp H \cdot\left[N_{G^{\prime}}\left(H \cap G^{\prime}\right)\right.$ : $\left.H \cap G^{\prime}\right]$.

Proof. If $T$ is an element of $N\left(G \mid H, G^{\prime}\right)$, then $H \cap G^{\prime}$ and $T^{*}\left(H \cap G^{\prime}\right)$ are of the same order in the cyclic group $T^{*}(H)$. Hence $T^{*}\left(H \cap G^{\prime}\right)=H \cap G^{\prime}$ and so we see that $N\left(G \mid H, G^{\prime}\right)$ is contained in $N_{G}\left(H \cap G^{\prime}\right)$. It is also easy to see the following facts (1) and (2).

(1) The mapping: $N_{G}(H) \times N\left(G \mid H, G^{\prime}\right) \rightarrow N\left(G \mid H, G^{\prime}\right), \quad(A, T) \mapsto A \cdot T$, is an action on the set $N\left(G \mid H, G^{\prime}\right)$.

(2) The mapping: $N\left(G \mid H, G^{\prime}\right) \times N_{G^{\prime}}\left(H \cap G^{\prime}\right) \rightarrow N\left(G \mid H, G^{\prime}\right),(T, B) \mapsto T \cdot B$, is an action on the set $N\left(G \mid H, G^{\prime}\right)$.

It follows from (1) and (2) that $N\left(G \mid H, G^{\prime}\right)$ has a double coset decomposition such as :

$$
N\left(G \mid H, G^{\prime}\right)=\bigcup_{\lambda} H \cdot T_{\lambda} \cdot N_{G^{\prime}}\left(H \cap G^{\prime}\right) \quad \text { (disjoint). }
$$

Thus in order to prove

$$
\# N\left(G \mid H, G^{\prime}\right)=\# H \cdot \#\{\lambda\} \cdot\left[N_{G^{\prime}}\left(H \cap G^{\prime}\right): H \cap G^{\prime}\right] \text {, }
$$

it suffices to show

$$
\begin{aligned}
& H \cdot T_{\lambda} \cdot B=H \cdot T_{\lambda} \cdot B^{\prime} \quad \text { if and only if } B^{\prime} \cdot B^{-1} \in H \cap G^{\prime}, \\
& \text { where } B \text { and } B^{\prime} \text { are elements of } N_{G^{\prime}}\left(H \cap G^{\prime}\right) .
\end{aligned}
$$

In fact, to prove the "if part", we assume that $B^{\prime} \cdot B^{-1}$ belongs to $H \cap G^{\prime}$. Since $T_{\lambda}$ belongs to $N_{G}\left(H \cap G^{\prime}\right)$, there is an element $B^{\prime \prime}$ in $H \cap G^{\prime}$ such that $T_{\lambda} \cdot B^{\prime} \cdot B^{-1}$ $=B^{\prime \prime} \cdot T_{\lambda}$. Then $H \cdot T_{\lambda} \cdot B^{\prime}=H \cdot T_{\lambda} \cdot B^{\prime} \cdot B^{-1} \cdot B=H \cdot B^{\prime \prime} \cdot T_{\lambda} \cdot B=H \cdot T_{\lambda} \cdot B$, as desired. To prove the "only-if part", we assume that $H \cdot T_{\lambda} \cdot B=H \cdot T_{\lambda} \cdot B^{\prime}$. Then $B^{\prime} \cdot B^{-1}$ belongs to $T_{\lambda}^{*}(H) \cap G^{\prime}=H \cap G^{\prime}$, as desired.

LEMMA 6. If $H^{\prime}$ is a nontrivial cyclic subgroup of $G^{\prime}$, then

$$
r_{*}\left(H^{\prime} ; G^{\prime}\right)=\sum_{H} r_{*}(H ; G),
$$

where $H$ ranges over the set $C Y\left(G \mid G^{\prime}, H^{\prime}\right)$ i.e. $\left\{H \in C Y(G) \mid H \cap G^{\prime}=H^{\prime}\right\}$.

Proof. It is trivial that $G^{\prime}$ satisfies $\left(E_{0}\right)$. In order to prove $\left(^{*}\right)$ we use the descending induction on $H^{\prime}$ in $C Y\left(G^{\prime}\right)$.

In the case where $H^{\prime}$ is maximal in $C Y\left(G^{\prime}\right)$, we have that

$$
r_{*}\left(H^{\prime} ; G^{\prime}\right)=r\left(H^{\prime}\right)=\sum_{H} r_{*}(H ; G),
$$

where $H$ ranges over the set $C Y\left(G \mid \supseteqq H^{\prime}\right)$, which now coincides with $C Y\left(G \mid G^{\prime}, H^{\prime}\right)$. 
In general cases, for the (fixed) $H^{\prime}$, we assume that $\left(^{*}\right.$ ) holds for each element $H_{\alpha}^{\prime}$ of $C Y\left(G^{\prime} \mid \supsetneq H^{\prime}\right)$ (instead of $H^{\prime}$ ). Then we see that

$$
\begin{aligned}
r_{*}\left(H^{\prime} ; G^{\prime}\right) & =r\left(H^{\prime}\right)-\sum_{H_{\alpha}^{\prime}} r_{*}\left(H_{\alpha}^{\prime} ; G^{\prime}\right) \\
& =r\left(H^{\prime}\right)-\sum_{H_{\alpha}^{\prime}{ }_{H}{ }_{\alpha \beta}} r_{*}\left(H_{\alpha \beta} ; G\right) \\
& =r\left(H^{\prime}\right)-\sum_{H_{r}} r_{*}\left(H_{\gamma} ; G\right)+\sum_{H} r_{*}(H ; G) \\
& =\sum_{H} r_{*}(H ; G),
\end{aligned}
$$

where $H_{\alpha}^{\prime}$ (resp. $\left.H_{\alpha \beta}, H_{\gamma}, H\right)$ ranges over the set $C Y\left(G^{\prime} \mid \supseteqq H^{\prime}\right)\left(\right.$ resp. $C Y\left(G \mid G^{\prime}, H_{\alpha}^{\prime}\right)$, $\left.C Y\left(G \mid \supseteqq H^{\prime}\right), C Y\left(G \mid G^{\prime}, H^{\prime}\right)\right)$.

Q.E.D.

Proof of Proposition 4. Let $H^{\prime}$ be an element of $C Y\left(G^{\prime}\right)$. Let $\left\{H_{\alpha}\right\}$ be a set of representatives of $G$-conjugacy classes of $C Y\left(G \mid G^{\prime}, H^{\prime}\right)$. This yields a decomposition :

$$
C Y\left(G \mid G^{\prime}, H^{\prime}\right)=\bigcup_{\alpha} C Y\left(G \mid G^{\prime}, H^{\prime}\right)_{\alpha} \quad \text { (disjoint), }
$$

where each $C Y\left(G \mid G^{\prime}, H^{\prime}\right)_{\alpha}$ denotes the set $\left\{T^{*}\left(H_{\alpha}\right) \mid T \in N\left(G \mid H_{\alpha}, G^{\prime}\right)\right\}$. Let $n_{\alpha}$ denote the integer $\sharp N\left(G \mid H_{\alpha}, G^{\prime}\right) / \sharp H_{\alpha} \cdot\left[N_{G^{\prime}}\left(H^{\prime}\right): H^{\prime}\right]$ (see Lemma 5). Then we have

$$
\begin{aligned}
\sharp C Y\left(G \mid G^{\prime}, H^{\prime}\right)_{\alpha} & =\left[N_{G^{\prime}}\left(H^{\prime}\right): H^{\prime}\right] \cdot n_{\alpha}\left(\sharp H_{\alpha} / \# N_{G}\left(H_{\alpha}\right)\right) \\
& =\left[N_{G^{\prime}}\left(H^{\prime}\right): H^{\prime}\right] \cdot n_{\alpha} /\left[N_{G}\left(H_{\alpha}\right): H_{\alpha}\right] .
\end{aligned}
$$

It follows from Lemma 6 that

$$
\begin{aligned}
r_{*}\left(H^{\prime} ; G^{\prime}\right) & =\sum_{\alpha} \sum_{H_{\alpha \beta}} r_{*}\left(H_{\alpha \beta} ; G\right) \\
& =\sum_{\alpha} \sharp C Y\left(G \mid G^{\prime}, H^{\prime}\right)_{\alpha} \cdot r_{*}\left(H_{\alpha} ; G\right) \\
& =\sum_{\alpha} l\left(H_{\alpha} ; G\right) \cdot n_{\alpha} \cdot\left[N_{G^{\prime}}\left(H^{\prime}\right): H^{\prime}\right],
\end{aligned}
$$

where $H_{\alpha \beta}$ ranges over the set $C Y\left(G \mid G^{\prime}, H^{\prime}\right)_{\alpha}$. Hence we conclude that

$$
l\left(H^{\prime} ; G^{\prime}\right)=\sum_{\alpha} l\left(H_{\alpha} ; G\right) \cdot n_{\alpha} .
$$

To this expression we apply our assumption that each $l\left(H_{\alpha} ; G\right)$ is a nonnegative integer. Then we see that $l\left(H^{\prime} ; G^{\prime}\right)$ is also a nonegative integer. This completes the proof of Proposition 4.

Remark. The above proof of Proposition 4 is purely "group theoretic". 


\section{$\S 2$. Automorphism groups of a Riemann surface of genus two as linear groups.}

2.1. We introduce another necessary condition in order to characterize $R(M, A G)$ 's (in the case $g=2$ ) by determining them.

Definition. Let $A$ be an element of $G L(g, C)$ of order $n>1$. We say that $A$ satisfies $(E)$ if there are integers $\nu_{1}, \cdots, \nu_{r}(r \geqq 0)$ which are prime to $n$ such that

$$
\operatorname{Tr}(A)=1+\sum_{i=1}^{r}\left\{\zeta_{n}^{\nu} i /\left(1-\zeta_{n}^{\nu} \imath\right)\right\},
$$

where $\zeta_{n}=\exp (2 \pi \sqrt{-1} / n)$. For a finite subgroup $G$ of $G L(g, C)$ we say that $G$ satisfies $(E)$ if each element $(\neq I)$ of $G$ satisfies $(E)$.

Remark. (i) If $A$ and $\nu_{1}, \cdots, \nu_{r}$ are as above, then $r=2-\left\{\operatorname{Tr}(A)+\operatorname{Tr}\left(A^{-1}\right)\right\}$ i.e. $r=r(A)$ (cf. [3], V. 2.9.).

(ii) If $G=R(M, A G)$ then $G$ satisfies $(E)$ by the Eichler trace formula (see e.g. [3], V. 2.9.).

The purpose of this section is to prove the following.

THEOREM 1. Let $G$ be a finite subgroup of $G L(2, C)$. Then the following two conditions are equivalent.

(1) There is a compact Riemann surface $M$ of genus two and an automorphism group $A G$ of $M$ such that $R(M, A G)$ is $G L(2, C)$-conjugate to $G$.

(2) $G$ satisfies the conditions $\left(R H_{+}\right)$and $(E)$.

To prove the theorem, we shall use the following properties on $\left(R H_{+}\right)$and (E) frequently but implicitly.

Remark. Let $G$ be a finite subgroup of $G L(g, C)$ which satisfies $\left(R H_{+}\right)$ (resp. $(E)$ ). We have the following.

(1) If $T$ is an element of $G L(g, C)$ then $T^{*}(G)$ is also satisfies $\left(R H_{+}\right)$ (resp. $(E))$.

(2) If $G^{\prime}$ is a subgroup of $G$ then $G^{\prime}$ also satisfies $\left(R H_{+}\right)$(resp. $(E)$ ).

2.2. Notation. We set the notation for later use.

Notation. Setting

$$
D(\alpha, \delta)=\left(\begin{array}{ll}
\alpha & 0 \\
0 & \delta
\end{array}\right), \quad B(\beta, \gamma)=\left(\begin{array}{ll}
0 & \beta \\
\gamma & 0
\end{array}\right),
$$

we define distinguished elements of $G L(2, C)$ as follows. 


$$
\begin{aligned}
& I=D(1,1), \quad J=D(-1,-1), \quad A_{2}=A(1,-1), \quad A_{3}=D\left(\zeta_{3}^{2}, \zeta_{3}\right), \\
& A_{4}=D\left(\zeta_{4}^{3}, \zeta_{4}\right), \quad A_{5}=D\left(\zeta_{5}^{2}, \zeta_{5}\right), \quad A_{6}=D\left(\zeta_{6}^{2}, \zeta_{6}\right), \quad A_{8}=D\left(\zeta_{8}^{3}, \zeta_{8}\right), \\
& B_{2}=B(1,1) \text { and } B_{4}=B(1,-1) .
\end{aligned}
$$

And we set

$$
S_{3}=\left(\begin{array}{ll}
\zeta_{8}^{3} / \sqrt{2} & \zeta_{8}^{7} / \sqrt{2} \\
\zeta_{8}^{5} / \sqrt{2} & \zeta_{8}^{5} / \sqrt{2}
\end{array}\right) .
$$

It is noted that $S_{3} \sim A_{3}$, where $\sim$ means the relation of $G L(2, C)$-conjugacy.

Notation-Lemma 2. We define distinguished subgroups $G(n, m)$ of $G L(2, \boldsymbol{C})$

\begin{tabular}{|c|c|c|}
\hline$G(1,2)=\langle I\rangle$ & $\mathfrak{B}_{1}$ & {$[1,2 ;-]$} \\
\hline$G(2,2)=\langle J\rangle$ & $\mathbb{S}_{2}$ & {$[2,0 ; 2,2,2,2,2,2]$} \\
\hline$G(2,4)=\left\langle A_{2}\right\rangle$ & $B_{2}$ & {$[2,1 ; 2,2]$} \\
\hline$G(3,12)=\left\langle A_{3}\right\rangle$ & $\left(S_{3}\right.$ & {$[3,0 ; 3,3,3,3]$} \\
\hline$G(4,4)=\left\langle J, A_{2}\right\rangle$ & $\mathfrak{D}_{2}$ & {$[4,0 ; 2,2,2,2,2]$} \\
\hline$G(4,8)=\left\langle A_{4}\right\rangle$ & $\mathbb{B}_{4}$ & {$[4,0 ; 2,2,4,4]$} \\
\hline$G(5,10)=\left\langle A_{5}\right\rangle$ & $\mathfrak{b}_{5}$ & {$[5,0 ; 5,5,5]$} \\
\hline$G(3 \cdot 2,12)=\left\langle A_{3}, B_{2}\right\rangle$ & $\mathfrak{D}_{3}$ & {$[6,0 ; 2,2,3,3]$} \\
\hline$G(6,12)=\left\langle J \cdot A_{3}\right\rangle$ & $\mathbb{S}_{6}$ & {$[6,0 ; 2,2,3,3]$} \\
\hline$G(6,24)=\left\langle A_{6}\right\rangle$ & $\mathbb{S}_{6}$ & {$[6,0 ; 3,6,6]$} \\
\hline$G(8,8)=\left\langle A_{4}, B_{2}\right\rangle$ & $\mathfrak{D}_{4}$ & {$[8,0 ; 2,2,2,4]$} \\
\hline$G(4 \cdot 2,48)=\left\langle A_{4}, B_{4}\right\rangle$ & $\langle 2,2,2\rangle$ & {$[8,0 ; 4,4,4]$} \\
\hline$G(8,48)=\left\langle A_{8}\right\rangle$ & $\left(s_{8}\right.$ & {$[8,0 ; 2,8,8]$} \\
\hline$G(10,10)=\left\langle J \cdot A_{5}\right\rangle$ & $\mathfrak{\wp}_{10}$ & {$[10,0 ; 2,5,10]$} \\
\hline$G(12,12)=\left\langle J \cdot A_{3}, B_{2}\right\rangle$ & $\mathfrak{D}_{6}$ & {$[12,0 ; 2,2,2,3]$} \\
\hline$G(2 \cdot 6,24)=\left\langle J, A_{6}\right\rangle$ & $\mathbb{B}_{2} \times \mathbb{B}_{6}$ & {$[12,0 ; 2,6,6]$} \\
\hline$G(4 \cdot 3,24)=\left\langle J \cdot A_{3}, B_{4}\right\rangle$ & $\langle 2,2,3\rangle$ & {$[12,0 ; 3,4,4]$} \\
\hline$G(16,48)=\left\langle A_{8}, B_{4}\right\rangle$ & $\langle-2,4 \mid 2\rangle$ & {$[16,0 ; 2,4,8]$} \\
\hline$G(24,24)=\left\langle A_{6}, B_{2}\right\rangle$ & $(4,6 \mid 2,2)$ & {$[24,0 ; 2,4,6]$} \\
\hline$G(24,48)=\left\langle A_{4}, S_{3}\right\rangle$ & $\langle 2,3,3\rangle$ & {$[24,0 ; 3,3,4]$} \\
\hline$G(48,48)=\left\langle A_{8}, S_{3}\right\rangle$ & $\langle-3,4 \mid 2\rangle$ & {$[48,0 ; 2,3,8]$} \\
\hline
\end{tabular}
as in the following table. And we list the group structure (using the symbols in [2]) and the RH data of each $G(n, m)$. We note that in general $G(n, m)$ is a subgroup of $G(m, m)$ such that $d(G(n, m))=d(G(m, m))(c f .(1.4))$. 
Proof. Cf. [2], Table 9.

(1) Putting $T=B_{4} \cdot A_{8}$ and $S=A_{8}$, we have that $T^{2}=I$ and $T \cdot S \cdot T=S^{3}$. This means that the symbol of $G(16,48)$ is $\langle-2,4 \mid 2\rangle$.

(2) Putting $R=B_{4}$ and $S=A_{3}$, we have that $R^{4}=S^{6}=(R \cdot S)^{2}=\left(R^{-1} S\right)^{2}=I$. This means that the symbol of $G(24,24)$ is $(4,6 \mid 2,2)$.

(3) Putting $R=J \cdot S_{3}$ and $S=S_{3}^{-1} \cdot B_{4}$, we have that $R^{3}=S^{3}=(R \cdot S)^{2}$. This means that the symbol of $G(24,48)$ is $\langle 2,3,3\rangle$.

(4) Putting $S=\left(A_{8} \cdot S_{3}\right)^{-1}$ and $T=S_{3} \cdot A_{8}^{3}$, we have that $S^{4}=(S \cdot T)^{3}$ and $T^{2}=I$. This means that the symbol of $G(48,48)$ is $\langle-3,4 \mid 2\rangle$.

(5) Considering the group structure, we are able to calculate $\operatorname{RH}(G(n, m))$ easily, so we omit the detail.

2.3. Here we prove a lemma on the normalizer of $G(n, m)$ in $G L(2, C)$. Before the statement, we set the notation.

Notation. (1) For a subgroup $G$ of $G L(2, \boldsymbol{C}), N_{G L}(G)$ (resp. $C_{G L}(G)$ ) denotes the normalizer (resp. centralizer) of $G$ in $G L(2, C)$.

(2) $Z G L=\{D(\alpha, \alpha) \mid \alpha \in C, \alpha \neq 0\}$, $D G L=\{D(\alpha, \delta) \mid \alpha, \delta \in C, \alpha \delta \neq 0\}$, $B G L=\{B(\beta, \gamma) \mid \beta, \gamma \in C, \beta \gamma \neq 0\}$.

Remark. It is easy to see the following.

(1) $C_{G L}(\langle D(\alpha, \delta)\rangle)=D G L$ if $\alpha \neq \delta$.

(2) $C_{G L}(\langle B(\beta, \gamma)\rangle) \cap D G L=Z G L$.

LEMMA 3. We have the following.

$$
\begin{aligned}
& N_{G L}(G(4,4))=N_{G L}(G(4,8))=D G L \cup B G L, \\
& N_{G L}(G(6,24))=D G L=C_{G L}(G(6,24)), \\
& N_{G L}(G(6,12))=N_{G L}(G(8,48))=N_{G L}(G(2 \cdot 6,24))=D G L \cup B G L, \\
& N_{G L}(G(12,12))=N_{G L}(G(4 \cdot 3,24))=G(24,24) \cdot Z G L, \\
& N_{G L}(G(4 \cdot 2,48))=N_{G L}(G(24,48))=G(48,48) \cdot Z G L .
\end{aligned}
$$

Proof. Considering the group structure, we see easily the desired facts. So we omit the details.

Q.E.D.

2.4. Lemmas. To prove the main part of Theorem 1, we shall prepare some lemmas such as

LEMMA 4. Let $G=\langle A\rangle$ be a finte cyclic subgroup of $G L(2, C)$ which satrsfies $\left(R H_{+}\right)$and $(E)$. Then $G$ is conjugate to some $G(n, m)($ in $(2.2))$.

Before giving its proof, we insert a sublemma on $R H(G)$. 
Sublemma. Let $G$ be a finte subgroup of $G L(2, C)$ which satisfies $\left(R H_{+}\right)$, say $R H(G)=\left[n, g_{0} ; n_{1}, \cdots, n_{r}\right]$. Assume $n_{r}=n$ (where $r \geqq 1$ ). Then $R H(G)$ is equal to one of the following.

$[2,0 ; 2,2,2,2,2,2], \quad[2,1 ; 2,2], \quad[3,0 ; 3,3,3,3], \quad[3,1 ; 3]$, $[4,0 ; 2,2,4,4],[5,0 ; 5,5,5],[6,0 ; 2,2,2,6],[6,0 ; 3,6,6]$, $[8,0 ; 2,8,8],[9,0 ; 3,3,9], \quad[10,0 ; 2,5,10], \quad[12,0 ; 2,4,12]$, $[18,0 ; 2,3,18]$.

Proof. Since $G$ satisfies $\left(R H_{+}\right)$, it follows from Proposition 1 in $\S 1$ that $R H(G)$ satisfies the relation:

$$
2 \cdot 2-2=n\left(2 \cdot g_{0}-2\right)+n \cdot \sum_{\imath=1}^{r}\left(1-\left(1 / n_{\imath}\right)\right) .
$$

On the other hand it is easy to find all the (desired type of) solutions of the relation $(R H)$ for a given integer $n$. Since now $n \leqq 84(2-1)$ (see (1.4)), we have done in principle.

Here we wish to show $n \leqq 18$, so suppose $n>18$. Then it is obvious that $g_{v}=0$ and $r \geqq 3$. If $r \geqq 4$ then

$$
2+2 n=n \sum_{\imath=1}^{r}\left(1-\left(1 / n_{\imath}\right)\right) \geqq 3(n / 2)+(n-1)
$$

and hence that $n \leqq 6$. If $r=3$ then

$$
2+2 n \geqq(n / 2)+(2 n / 3)+(n-1) \quad \text { i.e. } n \leqq 18 \text {, }
$$

since $[n, 0 ; 2,2, n]$ is not a solution. Thus we have a contradiction.

Q.E.D.

Proof of Lemma 4. Trivially it may be assumed that $\# A=n>1$. Using Jordan canonical forms, we may assume (cf. (2.1)) that $A=D\left(\zeta_{n}^{k}, \zeta_{n}^{l}\right)$, where $0 \leqq k, l<n$. If $k=0$ or $l=0$ then we may assume moreover that $l=1$, replacing $A$ by its some power or its some conjugate element if necessary. When $k \neq 0$ and $l \neq 0$, we may also assume $l=1$, if we show that $(l, n)=1$ or $(k, n)=1$. Suppose contrary that $(l, n)=m>1$ and $(k, n)>1$. Then the order of $A^{m^{\prime}}=$ $D\left(\zeta_{n}^{k m^{\prime}}, 1\right)$ is $m$, where $m^{\prime}=n / m$. Since now $A^{m^{\prime}}$ satisfies $(E), 2-2 \cdot R e\left(1+\zeta_{n}^{k m^{\prime}}\right)$ is a nonnegative integer. Hence $\zeta_{n}^{k m^{\prime}}=\zeta_{4}^{ \pm 1}, \zeta_{3}^{ \pm 1}$ or -1 . It is easy to see that any of $D\left(\zeta_{4}, 1\right)$ and $D\left(\zeta_{3}, 1\right)$ does not satisfy $(E)$. Hence $\zeta_{n}^{k m^{\prime}}=-1$ and $m=2$. Similarly we obtain that $(l, n)=(k, n)=2$, which is absurd, since $\# A$ is $n$.

Next we prove the lemma under an additional condition that $r(A)=0$. Then it follows from $(E)$ that $\operatorname{Tr}(A)=\zeta_{n}+\zeta_{n}^{k}=1$. From this it is easy to see that $n=6$ and $k=5$ and hence $G=G(6,12)$.

Assuming $r(A) \neq 0$, we examine the following cases $(1), \cdots,(10)$. We note then $R H(G)$ appears in the list of the Sublemma.

(1) Case: $n=2$. Then trivially $G$ equals to either $G(2,2)$ or $G(2,4)$.

(2) Case $: n=3$. Then obviously $D\left(\zeta_{3}^{k}, \zeta_{3}\right)(k=0,1)$ does not satisfy $(E)$. 
Hence $k=2$ i.e. $G=G(3,12)$ (if this case occurs).

(3) Case : $n=4$. Then obviously $D\left(\zeta_{4}^{k}, \zeta_{4}\right)(k=0,1)$ does not satisfy $(E)$ and $\left\langle D\left(\zeta_{4}^{2}, \zeta_{4}\right)\right\rangle$ does not satisfy $\left(R H_{+}\right)$. Hence $k=3$ i.e. $G=G(4,8)$.

(4) Case $: n=5$. Then $D\left(\zeta_{5}^{k}, \zeta_{5}\right)(k=0,1,4)$ does not satisfy $\left(E_{0}\right)$. Hence $k=2$ or $k=3$. From this $G \sim G(5,10)$.

(5) Case : $n=6$. Then it follows from (2) that $\left\langle A^{2}\right\rangle \sim G(3,12)$, since $\left\langle A^{2}\right\rangle$ also satisfies $\left(R H_{+}\right)$and $(E)$ (note $A^{2}=D\left(\zeta_{3}^{k}, \zeta_{3}\right)$ with $r\left(A^{2}\right) \neq 0$ ). Hence $k=2$ or $k=5$. Since $r\left(D\left(\zeta_{6}^{5}, \zeta_{6}\right)\right)=0$, we see that $k=2$ i.e. $G=G(6,24)$.

(6) Case : $n=8$. Then it follows as above that $\left\langle A^{2}\right\rangle \sim G(4,8)$. Hence $k=3$ or $k=7$. Since $D\left(\zeta_{8}^{7}, \zeta_{8}\right)$ does not satisfy $\left(E_{0}\right)$, we obtain that $k=3$ i.e. $G=G(8,48)$.

(7) Case : $n=9$. Then it follows as above that $\left\langle A^{3}\right\rangle \sim G(3,12)$ and hence $r\left(A^{3}\right)=4$. On the other hand since $\left\langle A^{3}\right\rangle$ is the unique subgroup of order 3 , it follows from $R H(G)=[9,0 ; 3,3,9]$ that $r\left(\left\langle A^{3}\right\rangle\right)=3+3+1$. This contradiction implies that this case does not occur.

(8) Case: $n=10$. Then $R H(G)=[10,0 ; 2,5,10]$ hence $r\left(\left\langle A^{5}\right\rangle\right)=5+0+1$. This means $A^{5}=J$ by (1). On the other hand $\left\langle A^{2}\right\rangle \sim\left\langle A_{5}\right\rangle$ by (4). Hence $G \sim$ $\left\langle J \cdot A_{5}\right\rangle$ i.e. $G \sim G(10,10)$.

(9) Case : $n=12$. Then $R H(G)=[12,0 ; 2,4,12]$ hence $r\left(\left\langle A^{6}\right\rangle\right)=6+3+1$. On the other hand $r\left(\left\langle A^{6}\right\rangle\right)=2$ or 6 by (1). This contradiction implies that this case does not occur.

(10) Case : $n=18$. Considering the 3-Sylow subgroup and (7), we see that this case does not occur.

This exhaustion (of cases) completes the proof.

Q.E.D.

COROLlary 5. Let $\langle B(\beta, \gamma)\rangle$ be a finite subgroup of $G L(2, C)$ whach satisfies $\left(R H_{+}\right)$and $(E)$. Then $\langle B(\beta, \gamma)\rangle$ is $D G L$-conjugate to $\left\langle B_{2}\right\rangle$ or $\left\langle B_{4}\right\rangle$.

Proof. Since $B(\beta, \gamma)^{2}=D(\beta \gamma, \beta \gamma)$, we obtain by Lemma 4 that $B(\beta, \gamma)^{2}=I$ or $J$. If $B(\beta, \gamma)^{2}=I$ (resp. $J$ ), then $D(\beta, 1)^{-1} \cdot B(\beta, \gamma) \cdot D(\beta, 1)=B_{2}$ (resp. $B_{4}$ ).

Q.E.D.

LEMMA 6. Let $G$ be a finite subgroup of $G L(2, C)$ which satısfies $\left(R H_{+}\right)$and $(E)$. Suppose that $\sharp G=2^{a}(a \geqq 0)$. Then $G$ is conjugate to some $G(n, m)($ in $(2.2))$.

Proof. We may assume that $G$ is not cyclic (and hence that $a \geqq 2$ ) by Lemma 4. To prove Lemma 6 we shall examine the following cases (1), $\cdots$, (4).

(1) Case : $a=2$. Since then $G$ is abelian, $G$ can be diagonalized simultaneously i.e. $G$ is conjugate to a subgroup in $D G L$ (by a result of linear algebra). Thus $G \sim G(4,4)$.

(2) Case : $a=3$. This case breaks into three subcases (see e.g. [2], Table 1.).

(a) Subcase: $G$ is abelian. Then we may assume (cf. (1)) that $G$ is contained in $D G L$. Hence the type of the abelian group $G$ is $(4,2)$. And it follows from Lemma 4 that $G$ contains $A_{4}$ and $A_{2}$. This is absurd, because $A_{4}^{-1} \cdot A_{2}=$ $D\left(\zeta_{4}, \zeta_{4}\right)$ does not satisfy $(E)$.

(b) Subcase : $G$ is dihedral. Then we may assume by Lemma 4 that $G=$ 
$\left\langle A_{4}, B\right\rangle$ where $\left(B \cdot A_{4}\right)^{2}=I$ and $\sharp B=2$. Since $B$ is an element of $N_{G L}\left(\left\langle A_{4}\right\rangle\right)$, it follows from (2.3) that $B$ belongs to $B G L$, and hence from Corollary 5 that $G \sim$ $\left\langle A_{4}, B_{2}\right\rangle$ i.e. $G \sim G(8,8)$.

(c) Subcase: $G$ is quaternion. Then we may assume that $G=\left\langle A_{4}, B\right\rangle$ where $B^{2}=\left(B \cdot A_{4}\right)^{2}=J$. It follows as above that $G \sim\left\langle A_{4}, B_{4}\right\rangle$ i.e. $G \sim G(4 \cdot 2,48)$.

(3) Case : $a=4$. Then $R H(G)=[16,0 ; 2,4,8]$, because it is the unique solution of $(R H)$ when $n=16$. This means that $G$ contains an element of order 8 . Hence we may assume by Lemma 4 that $G=\left\langle A_{8}, B\right\rangle$ for some element $B$. This case breaks into two subcases by (2.3).

(a) Subcase : $B \in D G L$. Then $G$ should be abelian and so contain an abelian group of type $(4,2)$, which is absurd by (2).

(b) Subcase: $B \in B G L$. Then it follows from Corollary 5 that $G \sim\left\langle A_{8}, B_{4}\right\rangle$ $=G(16,48)$ or $G \sim\left\langle A_{8}, B_{2}\right\rangle \sim\left\langle A_{8}, B\left(\zeta_{8}, \zeta_{8}^{7}\right)\right\rangle=G(16,48)$.

(4) Case : $a \geqq 5$. It is easy to see that $(R H)$ has no solutions when $n=32$. Hence our case does not occur, since any group of order $2^{a}(a \geqq 5)$ has a subgroup of order 32 .

Since we have considered all the possible cases, these complete the proof of Lemma 6.

LEMMA 7. Let $G$ be a finite subgroup of $G L(2, C)$ which satisfies $\left(R H_{+}\right)$and (E). Suppose $\# G=2^{a} \cdot 3^{b}(a \geqq 0, b \geqq 1)$. Then $G$ is conjugate to some $G(n, m)$ (in $(2.2)$ ).

Proof. By Lemma 4 and Lemma 6 we may assume that $G$ is not cyclic and that $a \leqq 4$. It is easy to see that $(R H)$ has a unique solution $[9,0 ; 3,3,9]$ when $n=9$. Hence if $b \geqq 2$ then we see that $G$ contains an element of order 9 (using a theorem of Sylow). This contradiction to Lemma 4 means that $b=1$. Moreover then, since $G$ is assumed to be not cyclic, we have $a \neq 0$. To prove Lemma 7 we shall examine the following cases $(1), \cdots,(4)$.

(1) Case : $a=1$. Since then $G$ must be dihedral, we may assume by lemma 4 that $G=\left\langle A_{3}, B\right\rangle$ where $\sharp B=2$. Then it follows from (2.3) and Corollary 5 that $G \sim\left\langle A_{3}, B_{2}\right\rangle$ i.e. $G \sim G(3 \cdot 2,12)$.

(2) Case : $a=2$. This case breaks into four subcases (see e.g. [2], Table 1.).

(a) Subcase: $G$ is (noncyclic) abelian. Then by Lemma 6 we may assume that $G$ contains $G(4,4)$ and hence that $G$ is of the form $\left\langle J, A_{2}, A\right\rangle$ where $\sharp A=3$. Then $A$ belongs to $D G L$ by (2.3). Since $\langle A\rangle \sim\left\langle A_{3}\right\rangle$ by Lemma 4 , we conclude that $A=A_{3}^{ \pm 1}$ and hence that $G=G(2 \cdot 6,24)$.

(b) Subcase : $G$ is dihedral. Then $G=\langle A, B\rangle$ where $\sharp A=6$ and $\sharp B=2$. By Lemma 4 we may assume that $A=J \cdot A_{3}$ or $A_{6}$. Considering (2.3), we see that $A=J \cdot A_{3}$ and $B \in B G L$. And it follows from Corollary 5 that $G \sim\left\langle J \cdot A_{3}, B_{2}\right\rangle$ i.e. $G \sim G(12,12)$.

(c) Subcase: $G$ is tetrahedral. If this case occur then we may assume that $G$ contains $G(4,4)$ as a normal subgroup (by Lemma 6). It follows from (2.3) that $G$ is of the form $\left\langle J, A_{2}, B\right\rangle$ where $B$ is an element of $B G L$ of order 3. This contradicts to Corollary 5 . 
(d) Subcase: $G$ is $Z S$-metacyclic. Then we have that $G=\langle A, B\rangle$ where $A^{3}=B^{2}=(A \cdot B)^{2}$. As in (b) we may assume that $A=J \cdot A_{3}$ and $B \in B G L$. Since $\# B=4$, it follows from Corollary 5 that $G \sim\left\langle J \cdot A_{3}, B_{4}\right\rangle$ i.e. $G \sim G(4 \cdot 3,24)$.

(3) Case : $a=3$. Then the group $G$ is solvable, hence $G$ contains a normal subgroup $N$ of order 12 or 8 . We shall examine these two subcases.

(a) Subcase: $\# N=12$. Let $A$ be an element of $G$ such that $A \notin N$. By (2) we may assume that $N=G(12,12), G(2 \cdot 6,24)$ or $G(4 \cdot 3,24)$.

(i) If $N=G(12,12$ ) (resp. $G(4 \cdot 3,24)$ ), then it follows from Lemma 3 that $A=B \cdot D(\alpha, \alpha)$ for some element $B$ of $G(24,24)$ and $\alpha \in C$. Since now $G(24,24)$ $=N \cup A_{2} \cdot N$, we may assume that $B=I$ or $B=A_{2}$. Then $A^{2}$ belongs to $Z G L$ and so $A^{2}=I$ or $A^{2}=J$ by Lemma 4. If $A^{2}=J$ then $A \cdot A_{3}$ is an element of order 12 of $G$, which is absurd by Lemma 4. If $A^{2}=I$ then $\alpha^{2}=1$ and hence $A$ belongs to $G(24,24)$. This implies that $G=G(24,24)$.

(ii) If $N=G(2 \cdot 6,24)$ then it follows from Lemma 3 that $A$ is an element of $D G L \cup B G L$. If $A$ belongs to $D G L$ then $G$ is abelian and hence $G$ contains an element of order 12 , which is absurd. If $A$ belongs to $B G L$ then we obtain by corollary 5 that $G \sim G(24,24)$ (note that $G(24,24)$ contains $B_{2}$ and $B_{4}$ ).

(b) Subcase: $\# N=8$. Let $A$ be an element of $G$ such that $\# A=3$. By Lemma 6 we may assume that $N=G(8,8)$ or $G(8,48)$ or $G(4 \cdot 2,48)$.

(i) If $N=G(8,8)$ or $G(8,48)$, then $\left\langle A_{4}, A\right\rangle$ is a subgroup of order 12 and so we have done by (a).

(ii) If $N=G(4 \cdot 2,48)$, then it follows from Lemma 3 that $A=B \cdot D(\alpha, \alpha)$ for some element $B$ of $G(48,48)$ and $\alpha \in C$. Since $A^{3}=I, B^{3}$ belongs to $Z G L$ and so $B^{3}=I$ or $B^{3}=J$ by Lemma 4 . Therefore in any case it follows from Lemma 4 that $\operatorname{det}(B)=1$. On the other hand $\operatorname{det}(A)=1$ since $A \sim A_{3}$ by Lemma 4. Thus $\operatorname{det}(D(\alpha, \alpha))=1$ i.e. $\alpha^{2}=1$, and hence in particular $D(\alpha, \alpha) \in G(48,48)$. Since $G(24,48)$ consists of elements of $G(48,48)$ such that their determinants are 1 , we conclude that $A$ belongs to $G(24,48)$ and so $G=G(24,48)$.

(4) Case : $a=4$. Then the group $G$ is solvable, hence $G$ contains a normal subgroup $N$ of order 24 or 16 . We shall examine these two subcases.

(a) Subcase : $\sharp N=24$. Let $A$ be an element of $G$ such that $A \notin N$. By (3) we may assume that $N=G(24,24)$ or $G(24,48)$.

(i) If $N=G(24,24)$, then $R H(N)=[24,0 ; 2,4,6]$, which contradicts to $R H(G)$ (if occurs). In fact $[48,0 ; 2,3,8]$ is the unique solution of $(R H)$ when $n=48$.

(ii) If $N=G(24,48)$, then it follows from Lemma 3 that $A=B \cdot D(\alpha, \alpha)$ for some element $B$ of $G(48,48)$ and $\alpha \in C$. Since now $G(48,48)=N \cup A_{8} \cdot N$, we may assume that $B=I$ or $B=A_{8}$. Then by Lemma 4 it is easy to see that $\alpha^{2}=1$. Thus we obtain that $A$ belongs to $G(48,48)$ and hence that $G=G(48,48)$.

(b) Subcase: $\sharp N=16$. By Lemma 6 we may assume that $N=G(16,48)$. If $A$ denotes an element of $G$ such that $\sharp A=3$, then $\left\langle A_{8}, A\right\rangle$ is a subgroup of order 24 and so $G \sim G(48,48)$ from (a).

This exhaustion (of cases) completes the proof.

LEMMA 8. Let $G$ be a finite subgroup of $G L(2, C)$ which satisfies $\left(R_{+}\right)$ and $(E)$. Suppose that $\# G$ is divisible by 5 . Then $G$ is conjugate to $G(5,10)$ or 
$G(10,10)$.

Proof. Considering that now $\# G \leqq 84(2-1)$, we see easily that $R H(G)$ is one of the following.

$$
\begin{array}{lll}
{[5,0 ; 5,5,5],} & {[10,0 ; 2,5,10],} & {[15,0 ; 3,3,5],} \\
{[20,0 ; 2,5,5],} & {[30,0 ; 2,3,10],} & {[40,0 ; 2,4,5] .}
\end{array}
$$

We wish to show that $G$ is cyclic, in which case we have done by Lemma 4, so we assume (moreover) that $G$ is not cyclic. Then $R H(G)$ should be one of the below three.

(a) If $\# G$ is 30 , then $G$ contains a (cyclic) subgroup of order 15 by a theorem of Hall, which is absurd by Lemma 4.

(b) If $\# G$ is 20 (resp. 40), then any 2-Sylow subgroup of $G$ must contain $J$ by Lemma 6 . Hence $G$ contains a cyclic subgroup of order 10 , which must be conjugate to $G(10,10)$ by Lemma 4 . This contradicts to the $R H$ data of $G$, since $R H(G(10,10))=[10,0 ; 2,5,10]$.

This completes the proof of Lemma 8.

\subsection{Proof of Theorem 1 .}

The implication: $(1) \Rightarrow(2)$ is already remarked (cf. (1.4), (2.1)).

To prove the converse, we assume that $G$ is a finite subgroup of $G L(2, C)$ which satisfies $\left(R H_{+}\right)$and $(E)$. Then the prime factors of $\# G$ occur among $\{2,3,5\}$ by Lemma 4 . Hence it follows from Lemma 6 , Lemma 7 and Lemma 8 that $G$ is conjugate to some $G(n, m)$ (in (2.2)).

Let $H C_{48}$ (resp. $H C_{24}, H C_{10}$ ) denote the hyperelliptic Riemann surface (of genus 2) which is defined by the equation $y^{2}=x\left(x^{4}-1\right)\left(\right.$ resp. $y^{2}=x^{6}+1, y^{2}=$ $\left.x^{5}+1\right)$. Using the differentials $x d x / y$ and $d x / y$, we define the representations $R:$ Aut $\left(H C_{u}\right) \rightarrow G L(2, C)$ as in (1.1) (where $\left.u=48,24,10\right)$. Then it is (classical and) easy to see that $R\left(H C_{u}\right.$, Aut $\left.\left(H C_{u}\right)\right)=G(u, u)$ (cf. e.g. [7]).

Since our $G(n, m)$ is contained in one of the above $G(u, u)$ 's, this completes the proof.

\section{REFERENCES}

[1] Accola, R.D.M., On the number of automorphisms of a closed Riemann surface, Trans. Amer. Math. Soc., 131 (1968) 398-408.

[2] Coxeter, H.S.M. And Moser, W.O.J., Generators and relations for discrete groups, New York-Heidelberg-Berlin: Springer Verlag 1980 (Fourth edition).

[3] FarkAS, H.M. AND KRA, I., Riemann surfaces. New York-Heidelberg-Berlin: Springer Verlag 1980.

[4] Guerrero, I., Holomorphic families of compact Riemann surfaces with automorphisms, Illinois J. of Math., 26 (1982) 212-225.

[5] Kuribayashi, I., On Certain curves of genus three with many automorphisms, Tsukuba J. Math., 6 (1982) 271-288.

[6] Kuribayashi, I., On automorphısms of prime order of a Riemann surface as 
matrices, manuscripta math., 44 (1983) 103-108.

17.] Wiman, A., Über die hyperelliptischen Kurven und diejenıgen vom Geschlecht $p=3$, welche eindeutige Transformationen in sich zulassen, Bihang till $\mathrm{K}$. Vet. Akad. Handlinger, 21 (1895), I 3-23.

\author{
Institute of Mathematics \\ UNIVERSITY OF TSUKUBA \\ IBARAKI 305, JAPAN
}

